\title{
Innate immune system response against environmental temperature changes as a dangerous abiotic factor
}

\author{
Alinejad T. ${ }^{1}$, Bhassu S. ${ }^{2 *}$, Mustafa F.B. ${ }^{3 *}$ and Othman R.Y. ${ }^{1}$ \\ ${ }^{1}$ Genetics and Molecular Biology Div., Institute of Biological Sciences, University of Malaya, Kuala Lumpur, Malaysia \\ ${ }^{2}$ Centre for Research in Biotechnology for Agriculture, CEBAR, University of Malaya, Kuala Lumpur, Malaysia \\ ${ }^{3}$ Department of Geography, Faculty of Arts and Social Sciences, University of Malaya, Kuala Lumpur, Malaysia \\ Received: 02/07/2020, Accepted: 04/08/2020, Available online: 22/10/2020 \\ *to whom all correspondence should be addressed: e-mail: firuza@um.edu.my; subhabhassu@um.edu.my \\ https://doi.org/10.30955/gnj.003394
}

\section{Graphical abstract}

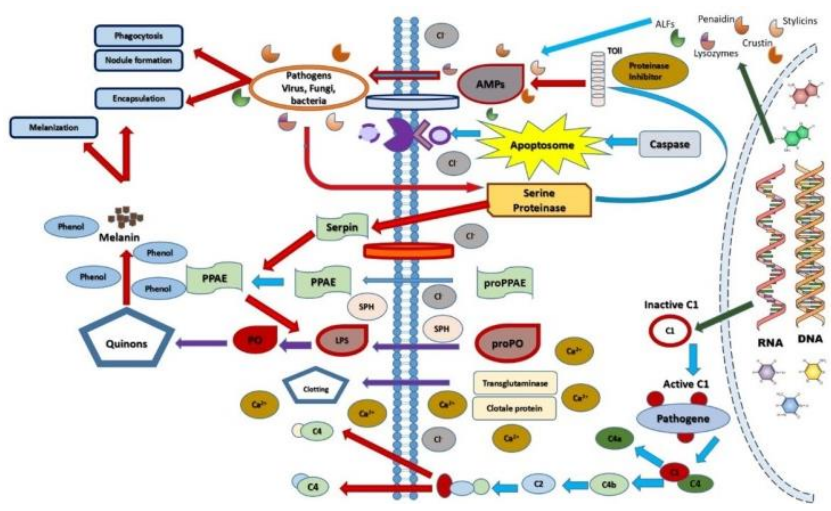

\begin{abstract}
Innate immune system is the first line response against environmental changes in invertebrate. It protects the animal from environmental changes such as temperature change, $\mathrm{PH}$ and salinity as well as pathogens such as bacteria and viruses through different biochemical pathways. In fact, the innate immune system relies on different biochemical reactions which are protecting the animal under adverse environmental circumstances. Among all of the environmental factors, temperature is a dangerous abiotic factor which affects organisms on its ecological level through infiltrating it's the molecular and cellular levels. Invertebrate could survive from a wide range of environmental effects and possesses innate immunity as its defense systems. This review paper aimed at presenting the main innate immune pathways that are activated against the most abiotic environmental changes. We reviewed fundamental aspects of invertebrates' defense process by focusing on the important innate immune pathways including: Pattern recognition receptors (PRRs), Antimicrobial peptide (AMP), Pro-PO activating system, Melanization Pathway, Lectin Pathway, Apoptosis Pathway, Plasma clotting protein.
\end{abstract}

Keywords: Innate immune system, invertebrates, environmental changes, temperature.

\section{Introduction}

Invertebrates and other arthropods share similar defense responses without the presence of immunoglobulins. Despite the absence of antigen-antibody specificity, the innate system could possess good identification and respond swiftly to incapacitate and eradicate pathogens. Primarily, the exoskeleton that shields the body structure is a natural physical barrier towards any types of pathogenic microorganisms (Pillai et al., 2010; Zulkapli et al., 2018). The existence of cuticle serves as a lining for foregut, hindgut of a prawn and its body surface. Moreover, gills could allow any exchange due to lack of epicuticle; whereas a gut present without outer lipid layer could allow the permeability. Crustaceans are enclosed with an open circulatory system that transports oxygen, hormones, nutrients, and cells via the hemolymph (Hosseini and Sharifi, 2019; Lorena Vazquez et al., 2009). Association of humoral and cellular defense mechanisms is observed during the hemocyte flow and helps to integrate with plasma cells. Humoral defense system involves the production and release of lectins, antimicrobial peptides (AMP) and prophenoloxidases (proPO) while cellular defense system includes hemocyte mediated responses for encapsulation and phagocytosis. The circulating fluids are known as crustacean hemolymph or blood comprise hemocytes that include fluids or plasma. The defense mechanism is stimulated based on the diverse arrangements of pathogen characteristics which are pointed at the surface level and involved in various immune responses. Upon intrusion of pathogens into the tissue, proteolytic pathways will be activated immediately in order to exterminate the pathogens (Ratcliffe et al., 1985). Firstly, it will be recognized and various effector cascades will be triggered such as antimicrobial proteins (Islam et al., 2020; Underhill and Ozinsky, 2002), clotting protein (Iwanaga and Morita, 1978; Iwanaga et al., 1978), lectin (Fujita, 2002), proPO system (Lorena Vazquez et al., 2009), encapsulation and phagocytosis (Bogdan et al., 2000). The proPO cascade could help activate other immune cascades such as the assembly of melanization, phagocytosis, encapsulation and nodule formation that are mediated 
through pathogen-associated molecular patterns (PAMPs) by crustacean proteins. The main question is that how, when and why a specific immune defense mechanism would be chosen in crustaceans among Pattern recognition receptors (PRRs), or pattern recognition proteins (PRPs), Apoptosis pathway, Melanization, Pro-PO activating system, Plasma Clotting protein, and Lectin Pathway (Akira et al., 2006). In crustaceans, the physiological barricade is the first hindrance to keep back any invading particles or pathogenic micro-organisms. When any damage, particles or pathogenic micro-organism is recognized, proteolytic pathways become active immediately (Haroon et al., 2018; Roth and Kurtz, 2009). This instantly activated pathway will eliminate the invaded particle. In second step hindrance, the coagulation cascade would be activated to avoid the loose of hemolymph. Then the proPO system will join the immune picture by stimulation of oxidative metabolites and melanin production. Prophenoloxidase is the most effective protein (Labbe and Little, 2009; Ogunyele et al., 2018). Its activation will stimulate other important procedures in the immune response. Phagocytosis and encapsulation and nodule formation will be activated by prophenoloxidase incitement. All of these pathways are interacting in a complex biochemical reaction that still has a lot of unveiled points. The complexity of the innate immune system reactions has been demonstrated in the Figure 1.

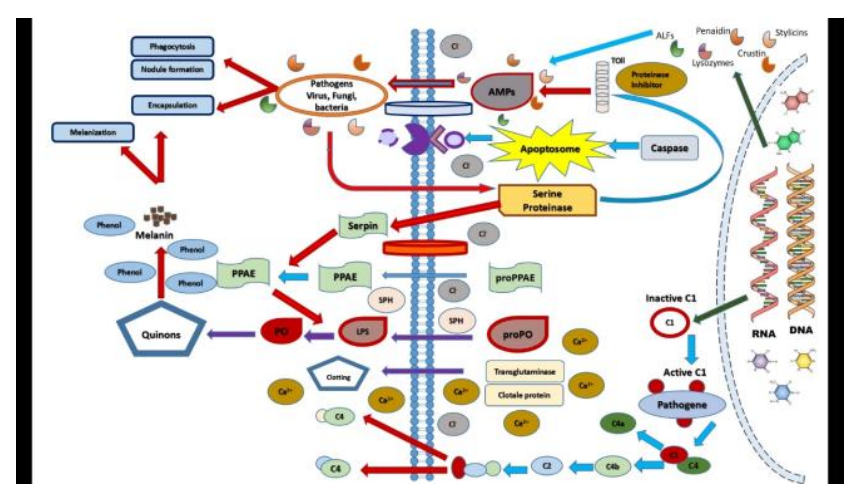

Figure 1. The figure is demonstrating the signal transduction and gene regulation of the innate immune system of crustacean. It shows the consequence of different pathways during the innate immune system activation under the adverse circumstances

\section{The effect of the temperature on the innate immune system molecular response}

The environmental temperature might have many fluctuations for many creatures throughout their lives. This effect may occur yearly, seasonally, or daily and usually does not remain constant. There is a question to ponder on, and that is that how an organism struggles with longterm or severe temperature changes (Asadullah et al., 2018; Hochachka and Somero, 2002; Youdeowei et al., 2019). Initial heat shock research investigates the mechanisms behind the response to critical heat stress by observing the heat shock in Drosophila. In this study, puffs characteristics had been studied in the salivary gland chromosomes of Drosophila (Ritossa, 1962). Later, it was understood that these studied chromosomal puffs were associated with RNA synthesis and heat shock protein expression. Since 1974 up till now, it is well known that heat shock protein family including (e.g, Hsp100, Hsp90, Hsp70, Hsp60, Hsp40 and small heat shock protein families) are regulated under "heat shock response" mechanisms in almost all organisms (Lindquist, 1986). The heat shock proteins are grouped by size or categorized by function. They are also famed as molecular chaperones because they are involved in protein folding. It has also been reported that they are preventing the formation of protein accumulation inside the cell (Parsell and Lindquist, 1993). Additionally, it have been reported that they have functions in ATP-independent' (small heat shock proteins) ATP-dependent (Hsp60, Hsp70) mechanisms (Fink, 1999). Initial studies revealed that the binding of the heat shock factor (HSF) to cis-regulatory heat shock element (HSE) regions induced heat shock regulatory networks. Furthermore, interaction between Hsp70 with HSF and can cause heat shock auto regulation or block it (Amina and Kamel, 2019; Morimoto and Santoro, 1998).

Researchers have approved that the heat stress response is a complicated mechanism. Research has also confirmed that the heat shock response has a variation in the initiation or termination timing or in the stress intensity. It furthermore depends on different types of Hsps in different organisms. It also may comprise from the induction of many other non Hsps genes (Brokordt et al., 2015; Junprung et al., 2017; Lindquist, 1986; Madeira, 2014; Ravaux et al., 2016).

The comparison between Drosophila and mussel Mytilus trossulus showed that heat in the Drosophila shock protein is synthesized during heat stress, but it will only be expressed after stress in mussel Mytilus trossulus. A range of HSPs proteins involved in response to heat were detected by faint SDS-PAGE bands in marine snails, yeast, and mussels. Hsps protein also maintains a fundamental influence for thermos-tolerance in yeast (Hoffmann, 2003).

Moreover, recent work on Drosophila and yeast has exposed no limitation in the binding target of the HSF to heat shock proteins. This confirmed that almost 3\% of genomic loci are available as binding target (Hahn et al., 2004). The recent interest in gene and mechanisms characterizations has risen by the development of genomic tools. The accessibility to the heat shock response studies by gene expression monitoring on a wide-ranging scale by high throughput technology and quantitative PCR has increased (Birch-Machin et al., 2005). Post research has shown that the level of change in gene and protein expression are not essentially correlated. Recent researches have confirmed that by some protein regulations. For instance (e.g., elongation factors) showed that these proteins are expressed at the translational level, but not in protein level or vice versa. This means that an increased in this gene expression generally does correlate with an increase in their protein expression (Suzuki et al., 2006). Moreover, additional research on Drosophila confirmed a parallel linkage between genomic and metabolomic profiles resulting in heat stress. 
Previously, most studies had focused on simple modeled organisms such as prokaryotes, Drosophila, and yeast. Recent studies; however, focused on the heat shock response in higher organisms (Hoffmann, 2003). Heat stress can cause many types of changes, fluctuations or variations in higher organisms or animals?

Higher aquatic organisms that are living in an aquatic habitat are widely exposed to a range of biophysical challenges. They are widely exposed to temperature fluctuations. These creatures may counteract these challenges by increasing or decreasing their body temperatures (Hofmann et al., 2005).

A noteworthy example is the $T$. funerals which was monitored in a study for 26 days from March-April. Nearly after half of the 26 days, the water temperature was midday low, but the body temperature was $27^{\circ} \mathrm{C}$. Its body temperature was high enough to induce Hsp70 and Hsp90 gene expressions (Tomanek and Somero, 1999).

Fascinatingly, in the similar heat stress condition, before midday $\left(2.5 \mathrm{~h}\right.$ at $\left.30^{\circ} \mathrm{C}\right), \mathrm{Hsp} 70, \mathrm{Hsp} 38, \mathrm{Hsp} 90$, and Hsp77 expression was increased and after $6 \mathrm{~h}, 30 \mathrm{~min} ., 6 \mathrm{~h}$, and $14 \mathrm{~h}$ it dropped to normal levels, this showed that T.funebrails can tolerate the stress response during the two low-temperature waves (Tomanek and Somero, 1999). In addition, the up-regulated HSPs levels that contribute to survival with reversible protein denaturation also exist following heat stress in the studied area. Reversible protein denaturation is the mechanism that handle the reversible denaturation through protein degradation (Hofmann et al., 2005).

Species-distributions borders are expected to change in response-to global warming. For example, in Monterey, California, southern species in the intertidal zone shifted northwards in the time span of 60 years (Sagarin et al., 1999). Environmental temperature is swiftly increasing both in domestic or aquatic habitats therefore understanding the aspects-of thermal tolerance physiology is essential. In the order to describe the impact that global warming and climate change may have on an organism (Stillman, 2003). It has been known that the upper and lower thermal tolerance limit (CTmax and CTmin) of an organism can be modified with acclimatizing to its surrounding temperature (Cuculescu et al., 1998). Thermal tolerance often depends on the thermal scale availability related to the conditions of the natural habitat. Stillman in 2003 used comparative approaches which demonstrated that during in created temperature, warm adapted species are less able to select their CTmax intolerance while coldadapted species are the opposite (Stillman, 2003). The geographical and latitudinal study may also be helpful for assessing the ecological consequences of climate change. It might have proper evidence in explaining the bases of an organism's distribution borders. A critical aspect of this study is the impact of global warming effects on species population in a latitude dependent approach. This aspect is assessed by thermal stress levels and the organisms, response in their natural habitat. Seasonal temperature conditions influence a thermal phenotype (Hopkin et al., 2006). It would be insightful to know the level of gradual acclimatization between seasons in natural habitats. There is evidence which shows that the thermal phenotype varies at both molecular and physiological levels (Dietz, 1994). For instance, in crab C. maenaas and $C$. pagurus, the maximum thermal tolerance was significantly lower in winter than it was in summer (Cuculescu et al., 1998).

There are some studies on the recovery from temperature fluctuation of intertidal specious such as mussel $M$. trossulus and crab, Petrolisthes cinctipes. Studies on the heat shock response in these species have confirmed the immersion of $\mathrm{Hsp} 70$ and $\mathrm{Hsp90}$ in reversible protein denaturation (Stillman, 2003). In this stage, a question arises that, what other mechanisms or genes either than Hsps family genes might be up or down-regulated in response to the thermal stress? And how do these changes may vary over time? And what is the immune system response?

\section{Pattern recognition receptors (PRRs)}

In this innate immune system mechanism, the invading microorganism would be recognized by germline-encoded RRs (Akira et al., 2006). The pattern recognition receptors will bind to the first available pathogen-associated molecular patterns (PAMPs) on the surface of the microorganism. Each PRRs specifically recognizes specific AMPs and activates signaling pathways of the immune responses. It will be active phagocytosis, encapsulation, nodule formation depending on the PAMPs or may be active AMPs synthesis (Medzhitov R and C., 2000).

\section{Antimicrobial peptide (AMP)}

AMPs could normalize and/or destroy various types of invading pathogens including Gram-positive, Gramnegative, fungal, viral and protozoans. It is reported in numerous species ranging from plants, invertebrate to vertebrates. It is present as a small cationic protein which has features of antimicrobial response and enhanced immunity by functioning as an immunomodulator. It was also best described as a candidate for development of novel antibiotics and also in the development of therapeutic agents. In shrimp, numerous types of AMPs have been characterized such as crustins, antilipopolysaccharides factor, penaeidins, hemocyanin, histones and many more. The two well-known AMP is well described in $M$. rosenbergii which is known as cousin and anti-lipopolysaccharides factor (Rosa et al., 2010).

Crustin is one of the antimicrobial peptides that is classified as a cationic cysteine-rich antibacterial polypeptide in crustaceans. Crustin which is the homolog of 'caring' was first identified inshore crab, Carcinus maenas as an $11.5 \mathrm{kDa}$ peptide (Relf et al., 1999). Crustins were isolated from a broad range of crustaceans which include Litopenaeus vannamei, Litopenaues setiferus (Bartlett et al., 2002), Panulirus argu (Stoss et al., 2004), Penaues monodon (Supungul et al., 2004), Marsupenaeus japonicas (Rattanachai et al., 2004), Homarus gammarus (Hauton et al., 2006), Fenneropenaeus chinensis (Zhang et al., 2007), Carcinus maenas (Brockton et al., 2007), Pacifastacus leniusculus (Jiravanichpaisal et al., 2007), Litopenaeus 
schmitti, Farfantepenaeus brasiliensis, Farfantepenaeus paulensis (Rosa et al., 2007), Farfantepenaeus subtilis (Rosa et al., 2007), Fenneropenaeus indicus (Antony et al., 2011), M. rosenbergii (Arockiaraj et al., 2013).

The term crustin was first coined to define the mRNA transcript found in L. vannamei and $L$. setiferus (Bartlett and Cuthbertson, 2002). Crustins are cationic, cysteine-rich AMPs with an isoelectric point ranging from 7.0 to 8.7 with a molecular weight ranging between 7 and $14 \mathrm{kDa}$ with one whey acidic protein (WAP) domain at the carboxyl terminus (Bartlett et al., 2002). This domain consists of tightly packed 8 cysteine residues in a conserved architecture which is known as a four-disulfide core (4 DSC). The WAP domain containing proteins are widely distributed from invertebrates to vertebrates. The term 'WAP' is given to a family of proteins that are derived from whey fraction of mammalian milk, and it comprises of two WAP domains with 50 amino acids (Ranganathan et al., 1999). The WAP domain in mammals includes trapping, elafin, and antileukoproteinase. It is one of the conserved points among many species and few crustins mainly in shrimp (Bartlett et al., 2002).

The molecular assemblies between the signal sequence and WAP domain vary in crustin, yet it is substantiated by its unique molecular architecture. The cysteine rich region lies in between the signal sequence ( $\mathrm{N}$-terminus) and the WAP domain (C-terminus) for Type I crustins which are present in crabs (Brockton et al., 2007; Imjongjirak et al., 2009) crayfish (Jiravanichpaisal et al., 2007), shrimp (Sun et al., 2010), freshwater prawn (Dai et al., 2009) and lobsters (Christie et al., 2007) and other crustins in the Pleocyemata family. This type of crustin was categorized under Type I since it resembles similar domain organizations as a carcinogen (Relf et al., 1999). Likewise, Type II crustin exists with both long glycine-rich and cysteine regions within the signal sequence ( $\mathrm{N}$-terminus) and a WAP domain (Cterminus) which are present mainly in crayfish (Jiravanichpaisal et al., 2007) and shrimp (Rattanachai et al., 2004). The Type III crustin is also known as a single whey domain (SWD), chelonianin-like protein or antileukoproteinase-like proteins. It is a peptide that lacks both cysteine and glycine-rich regions, but it is substituted with proline or arginine region between the signal sequence and WAP domain. So far only few Type III crustins were identified in crayfish and shrimp (Amparyup et al., 2008).

$M$. rosenbergii crustin carries a signal sequence and WAP domain along with proline and arginine-rich regions. It was also classified as a type III crustin (Arockiaraj et al., 2013). It also has 12 conserved cysteine residues that were previously observed in many other crustins as well as antimicrobial peptides like a carcinogen. The conserved residues were predicted to form six disulphide bonds in the tertiary structure. This WAP domain shows a wide range of functions including antiproteinase and antimicrobial activities. Moreover, the amino acid residue of protease inhibitors, methionine, which is located near to the second cysteine in WAP domain is replaced by cationic and hydrophobic amino acids in crustin WAP. This alteration turns the protein to be amphipathic and permits insertion of the protein into the outer layer of microbes.

Crustin as an AMP effector was reported to carry antibacterial activities especially in Gram-positive bacteria (Arockiaraj et al., 2013). Mr crustin could exhibit its response towards infectious hypodermal and hematopoietic necrosis virus (IHHNV) and white spot syndrome virus (WSSV) and bacteria Aeromonas hydrophila (Gram-negative) and Enterococcus faecium (Grampositive). Besides, the recombinant crustin also shows good response towards both Gram positive and Gram negative bacteria by distinguishing the pathogens (Arockiaraj et al., 2013). The first identified crustin from $C$. maenas has the ability to encounter the growth of Grampositive bacteria that include Aerococcus viridans var tomato, two strains of marine Planococcus spp. and a salttolerant strain of Micrococcus luteus (Relf et al., 1999). The recombinant crustin of $F$. chinesis also aims at Grampositive bacteria that include Staphylococcus aureus, $M$. luteus and 3 other bacilli (Zhang et al., 2007). One of the recombinant crustins of $P$. monodon highly inhibits the activity against Streptococcus iniae and $S$. aureus but not towards $A$. viridans var homari and $M$. luteus (Supungul et al., 2008). Nevertheless, Type II crustin identified from $P$. monodon indicates robust antibacterial activity against both Gram-positive and Gram-negative bacteria that include A. viridans var homari, Escherichia coli 363 and Vibrio harveyi (Amparyup et al., 2008).

On the other hand, anti-lipopolysaccharides factor (ALF) is one of the important conserved AMP found in crustaceans. Initially, ALF was known as anti-lipopolysaccharide LPS factor as it was recognized as an inhibitor factor of LPSmediated hemolymph coagulation in Limulus polyphemus and Tachypleus tridentatus (Tanaka et al., 1982). ALF was further identified and cloned in many other crustacean species such as Litopenaues setiferus (Gross et al., 2001; Li et al., 2014), Penaeus monodon (Supungul et al., 2002), Macrobrachium olfersi, Farfantepenaeus paulensis and Litopenaeus schmitti (Rosa et al., 2007), Macrobrachium rosenbergii (Jesu et al., 2012; Liu et al., 2014; Ren et al., 2012), Macrobrachium nipponense (Wang et al., 2015), Fenneropenaeus chinesis (Liu et al., 2005, Tang et al., 2014), Litopenaeus vannamei (Vega et al., 2008), Marsupenaeus japonicas (Hiroki Nagoshi et al., 2006; Mekata et al., 2005), Pacifastacus leniusculus (Liu et al., 2006), and Scylla paramamosain (Imjongjirak et al., 2009), Eriocheir sinensis (Zhao et al., 2009), Scylla serrata (Ko et al., 2007), Procambarus clarkii (Li et al., 2009) and Portunus trituberculatus (Yue et al., 2010).

Moreover, multiple isoforms also have been found in the many types of other crustacean's species. To date, seven forms of ALF genes were identified from $M$. rosenbergii (Arockiaraja et al., 2011). Five forms of ALF genes were reported from the two genomic loci from $P$. monodon that are actively involved in defense mechanisms against various types of pathogen infections (Antony et al., 2011). Portunus trituberculatus has four types of ALF genes (Yue et al., 2010). 
ALF is classified as a single domain AMP that has an LPS domain with a signal peptide. The LPS has two conserved cysteine residues that form the disulfide bonds. The deduced ALF from $M$. rosenbergii shows the conserved structure of the signal peptide, LPS binding domain and two cysteine residues (Arockiaraj et al., 2014). According to Yang et al. (1992), the second and third sheet of $\beta$-sheet is enveloped by the LPS domain along with the four antiparallel $\beta$-sheets. The structure of Macrobrachium rosenbergii showed $5 \alpha$-helices and $4 \beta$-sheet along with two conserved cysteine residues in $\beta$-sheet that forms disulfide bonds in a hairpin loop (Jesu Costa Ferreira et al., 2012) This similar pattern was also observed in ALF from $E$. sinensis (Wang et al., 2011). The pl value of ALF and LPS binding domain of $M$. rosenbergii falls in a range of 5.00 to 10.00 among crustaceans (Ren et al., 2012). Basically, ALF is a basic protein that is responsible for binding and neutralizing LPS. The basic ALF shows antibacterial activity, while the acidic ALF may show antiviral or antifungal activity (Ren et al., 2012). Similar results were obtained for E. chinesis, M. japonicas, Litopenaeus stylirostris (Zhang et al., 2010).

ALFs were reported to be involved in immune defense mechanisms in a wide range of pathogenic infections including bacteria, virus, fungi or yeast. Generally, ALF can bind to the liposaccharides (LPS) on the pathogens (Imjongjirak et al., 2007). MrALF shows it response against white spot syndrome baculovirus (WSBV), white spot syndrome virus (WSSV), Aeromonas hydrophila, Escherichia coli, Vibrio anguillarum, and Staphylococcus aureus. It is reported that cationic and hydrophobic properties of AMPs allow the interaction and insertion into the anionic cell walls and phospholipid membranes of the pathogens towards antimicrobial action (Morita et al., 1985; Oren et al., 1998). The ALF from Portunus trituberculatus increases upon Vibrio alginolyticus challenge. In Scylla paramamosain an ALF had demonstrated antimicrobial activity against Vibrio (Imjongjirak et al., 2011). While, the ALF in Pacifastacus leniusculus shows antiviral activity upon challenge with the white spot syndrome virus (WSSV) (Lu et al., 2006). The ALF from Penaues monodon was shown to be up-regulated upon Vibrio harveyi challenge (Tharntada et al., 2008).

Besides that, the functional property was indicated by recombinant ALF with LPS domain through various types of Gram-negative and Gram-positive bacteria (Liu et al., 2014). The recombinant ALF from M. rosenbergii could show antibacterial activity towards Aeromonas hydrophila, Vibrio cholera, and Escherichia coli. Moreover, it could also bind to both bacterial types that were studied including LPS of $E$. coli, and lipoteichoic acids of $B$. subtilis and $S$. faecalis. Meanwhile, (Arockiaraj et al., 2014) reported that MrALF shows a high antimicrobial response to almost more than five types of bacteria which were tested. Moreover, the bactericidal test after 24 hours also indicates that MrALF could kill all the bacteria. A similar type of activity was also shown by recombinant ALF protein from Scylla paramamosain, Penaeus monodon and Pacifastacus leniusculus (Somboonwiwat et al., 2008). The recombinant
ALF from Penaeus monodon has successfully decreased the replication of WSSV (Tharntada et al., 2009). This indicates the participation of MrALF as a part of defense mechanism during pathogen attack.

\section{Prophenoloxidase pathway}

The prophenoloxidase protein is the active form of pro-Po system and is the main domain protein of Pro-PO activating system. Pro-PO is synthesized and localized in crustacean's granules hemocyte. Then it triggers the pattern recognition protein and migrates to plasma by exocytosis process (Amparyup et al., 2013). The pro-Po system contains several protein complexes that will participate in melanin formation, cytotoxic reaction, cell adhesion, encapsulation, Prophenoloxidase, and phagocytosis (Söderhäll and Cerenius, 1998). Each crustacean species has a specific composition of the zymogens of either protease cascade, pattern recognition proteins or serine protease and proPO. The complex of pro-PO and LPS will bind to the microbial cell wall components and induce activation of serine protease zymogens in the pro-PO system (Amparyup et al., 2012). The most triggering molecules to pro-Po are LPS binding protein which is found in crayfish pro-PO system. In an in vitro study, the cleavage of pro-PO activating serine proteases showed antimicrobial activities which suggest that pro-PO activation with serine protease may have a dual function (Gupta et al., 2005).

A humoral response that plays a key role in antigen recognition and neutralization in a wide range of host models is known as prophenoloxidase (proPO) (Cerenius et al., 2008; Söderhäll and Cerenius, 1998). The proPO system was also identified as a center of the defense mechanism characterized in many organisms including arthropods, ascidians, cephalochordate, molluscs, and annelids (Theopold et al., 2004). A segment of complex enzyme cascade could activate $\mathrm{PO}$ along with $\mathrm{Ca}^{2+}$ ions that oxidizes phenols into quinone and further catalyzes it into melanin cascade for encapsulation, wound healing and for antimicrobial activity (Arockiaraja et al., 2011). Various types of microorganisms or parasites may activate the proPO cascade, especially the large size pathogen. This type of pathogen encapsulation and further melanization appears on the host system by the action of phenoloxidase (Arockiaraj et al., 2012; Söderhall et al., 2003). The proPO involves mainly in defense reactions, wound healing and cuticular hardening processes (Arockiaraj et al., 2012). The activation system of proPO can also be stimulated by very low quantities of microbial cell components such as $\beta-1,3-$ glucans, lipopolysaccharide (LPS), and peptidoglycan (PG) (Arockiaraj et al., 2012). These intruders could indirectly or directly induce the granulocytes via enzyme activating cascades to produce phenoloxidase (PO) (Arockiaraj et al., 2013).

The first cloned and purified proPO was from Pacifastacus leniusculus, a freshwater signal crayfish (AsPAN et al., 1995). Following which many other proPO genes were isolated and characterized from a wide range of species including Drosophila melanogaster (Fujimoto et al., 1995), Manduca sexta (Hall et al., 1995), Anopheles gambiae 
(Christophides et al., 2002), Scylla serrata (Ko et al., 2007), Anopheles stephensi (Cui et al., 2000), Aedes aegypti (Taft et al., 2001), Bombyx mori (Asano et al., 2001), Armigeres subalbatus (Huang et al., 2001), Litopenaeus vannamei (Liu et al., 2004), Penaeus monodon (Nayak et al., 2010), Homarus gammarus (Hauton et al., 2005), Macrobrachium rosenbergii (Arockiaraj et al., 2012). Upon infection, one of the major enzymes that are stimulated is PO via proPO activation system. This takes place upon stimulation by components of the pathogen-associated molecular pattern (PAMP). The activation of proPO cascade may involve serine protease breaks, to trigger the zymogen into active $\mathrm{PO}$. The PO is a copper-dependent enzyme that acts as a terminal enzyme with two functions. PO can catalyze monophenols into o-hydroxylation (cresolase activity) and oxidation of phenols into quinones (Sugumaran and Nellaiappan, 1996). Therefore, PO can convert tyrosine to dihydroxyphenylalanine (DOPA) and also DOPA to DOPAquinone along with non-specific pathways between neighboring molecules for melanin formation. Moreover, the $\mathrm{PO}$ is also able to regulate the negative stimulus to the host through reactive oxygen intermediates (ROI) and reactive nitrogen intermediates (RNI) (Cerenius and Soderhall, 2004; Ko et al., 2007).

The first MrproPO (MrproPO1) was cloned by (Lu et al., 2006) with six histidine residues and a thiol ester like motif. This peptide has similar structural formation as Penaues monodon, $P$. leniusculus, and $H$. gammarus. Furthermore, (Lu et al., 2006) also had reported proPO (MrproPO2) from $M$. rosenbergii with six histidine residues within two copper binding sites, thiol esters like motif, a proteolytic activation site, and a conserved c-terminal region. The proPO activating enzyme III (MrproPO3) is a conserved coppercontaining enzyme that was isolated and characterized in M. rosenbergii (Arockiaraj et al., 2012). The amino acid sequence has a characteristic feature of the clip-serine protease (clip-SP) that comprises of histidine active sites at $\mathrm{N}$ - and $\mathrm{C}$ - terminal, signal peptide and a domain from serine proteases trypsin family. This clip also shows conserved catalytic amino acid that is important for the stabilization of the catalytic site in the three-dimensional structure. The clip-SP is an important molecule that plays important roles in immune regulation and embryonic development. This sequence characteristic was also found in another proPO activating enzyme such as $C$. sapidus and $P$. monodon. In addition, another MrproPO (MrproPO4) was also found by (Arockiaraja et al., 2011) with two copper binding sites ( $\mathrm{CuA}$ and $\mathrm{CuB}$ ) that have hemocyanin features, a signal sequence that is important in secretion at endoplasmic reticulum site and thiol-ester like motif. Hemocyanin has a wide range of physiological processes such as osmoregulation, molt cycle, exoskeleton arrangement and protein storage. The similar structural study was also reported in Manduca sexta (Li et al., 2009). Besides that, the tertiary structure also indicates tyrosine signature that is postulated to bind to proPO and activate the melanin synthesis (Li et al., 2009). In addition, the copper binding sites with conserved histidine residues were found in most insects and crustaceans that are responsible for melanization, encapsulation and sclerotization mechanism (Hughes, 1999).

MrproPO1 gene expression and PO activity were measured using $\mathrm{CpG}$ oligodeoxynucleotide. This has clearly shown that the proPO system can be activated through CpG oligodeoxynucleotide via protein kinase $C$ signaling pathway. This supports their previous study as the challenge was carried out with Aeromonas veronii and Lactococcus garvieae. As a whole, the proPO system can be triggered by the stimulation of bacterial challenge, bacterin or oligodeoxynucleotide stimulation (Lu et al., 2006). Meanwhile, the expression of MrproPO2 was studied based on five different molting stages of $\mathrm{Mr}$ (Liu et al., 2006). The gene expression of proPO in the postmoult stage showed a gradual increase and further reached the higher expression levels during the later stage of the postmoult stage. The proPO expression declined sharply in the intermoult stage, and the lowest level was observed in the premoult stage. Therefore, it was concluded to be an immunomodulatory system (Liu et al., 2006). MrproPO3 shows its responses against IHHNV (Arockiaraja et al., 2012) while MrproPO4 shows responses towards a wide range of pathogens including WSBV, MrNV, A. hydrophila and V. Harvey (J et al., 2013)i (Arockiaraj et al., 2013). This indicates that MrproPO is actively involved in the defense mechanism of innate immunity in an invertebrate. The PO activity of $\mathrm{MrproPO} 3$ is not similar to gene expression study that might be because of its complex activity at different phases of the host-pathogen interaction. MrproPO4 shows a robust association between gene expression and enzymatic activity that might be because of its involvement of various phenoloxidases. A similar mechanism was also suggested by (Adachi et al., 2003).

\section{Melanization pathway}

Melanin is synthesized by the proPO-activating system and is an enzymatic cascade which is involved in several enzymes (Gorman MJ et al., 2008). This protein is the main substance for melanization which an important immune defense pathway in crustaceans is. The melanization is a prominent immune response which involves the synthesis of melanin to encapsulate pathogens. The process of melanization is a complex process which starts from proPOactivating system binding to microbial cell wall components. This complex will activate the stimulation of the SP cascade which promotes proteolysis to convert proPO into active PO. The active PO oxidase phenols into quinines which can non-specifically crosslink nearest molecules to form melanin. To date, several genes associated with the shrimp pro-PO activating system are identified and characterized (Jang et al., 2011).

\section{Lectin pathway}

Invertebrates have a well-developed defense mechanism to detect and exterminate any opportunistic microorganism or potential pathogens. Anciently diverse conserved molecules establish their non-self- recognition system through pathogen-associated molecular patterns (PAMPs). In response to this architecture, a family of proteins that were termed as pattern recognition proteins 
(PRPs) will be exerted by the immune system to initiate the downstream mechanisms. A well-studied protein, the lectin is a functional precursor of antibodies which recognizes the signature molecules of non-self-particles via PAMP (Kilpatrick, 2002). Lectins are organized in a broad spectrum of protein units, and every architecture shows different molecular mass depending on the species and the molecular assemblies. Lectins are proteins or glycoproteins. They normally do not have catalytic activity. They are mostly recognized while they don't have any covalent bind to sugar moities. Thus they agglutinate cell by binding to glycoproteins or glycoconjugates to the cell surface (Agundis et al., 2000). Therefore, they are considered as significant pattern recognition proteins in crustacean innate immunity. They also play important roles in non-self-recognition and clearance of invading microorganisms. They are existing in circulating fluids, either as cell surface receptors or soluble proteins (Marques and Barracco, 2000). Different types of lectins have been diagnosed in a crustacean. Lectin1, lectin2, lectin3, I3ctin4 and C-type lectins are the most recognized lectins in crustaceans. C-type lectins are the largest group of immune function that is mostly found in crustacean hepatopancreas (Zhang et al., 2007). A large number of lectins have been purified and characterized from crustacean hemolymph (Pereyra et al., 2004). In crustaceans, lectins have been reported to contribute to innate immune responses, including prophenoloxidase activation, encapsulation1.nodule formation of hemocyte, opsonin formation, antibacterial activity, and antifungal activity and may also be contributing to injury healing (Smith et al., 2003).

It is reported that lectin serves a decisive role for both physiological and pathological processes with exclusive interactions between intricate carbohydrates that include glycoproteins, glycolipids, polysaccharides or proteoglycans (Vázquez-Mendoza et al., 2013). Likewise, some lectins respond only to certain types of carbohydrate forms that encompass the whole sugar molecule, a portion of the molecule or the glycosidic linkages (Sharon and Lis, 1998). Generally, lectins are described to be activated based on the conserved sugar binding activity towards the $\mathrm{N}$ - or O-acetylated sugar residues, such as $\mathrm{N}$-acetylglucosamine (GlcNAc), N-acetyl- galactosamine (GalNAc), $\mathrm{N}$-acetyl-neuraminic acid, or O-acetylated sialic acid in decapod (Lorena Vazquez et al., 2009) Several activities take place during these multifaceted interactions such as the embryonic development, intracellular-trafficking, cellcell and cell-matrix recognition, cell homing, protein synthesis and transport, signal transduction, endocytosis, phagocytosis and inflammation (McGreal et al., 2004).

To date, many lectins are recognized and studied in their molecular configuration. As non-self- recognition molecules, lectins show a high abundance of activity in domain assemblies, sugar substrates, and tissue distribution and even expression upon pathogen challenge to describe the potential functional roles in immunological assays. Lectins are highly heterogeneous molecules that are categorized based on their structures, motifs, and functions. The motifs are known as carbohydrate recognition domains (CRD) that show the disparities between the structures. The Animal Lectins Homepage (http://www.imperial.ac.uk/ research/animal lectins/default) declared that animal lectins are termed into 13 different groups namely the C-type, F-type, M-type, L-type, P-type, I-type, R-type, F-box lectins, chitinase-like lectins, ficolins, calnexin, galectins, and intelectins. The intracellular lectins were reported in protein sorting and trafficking whereas extracellular lectins mainly were shown to function in cell signaling and pathogen recognition (Kilpatrick, 2002). Even though repertoires of lectin show a large divergence, almost 7 types of lectins were identified in the GenBank that are known as C-type, M-type, L-type, P-type, fibrinogen-like domain lectins, galectins and calnexin/calreticulin. Sequence distinction in CRD indicates diverse interactions between sugars that are known as mannose-binding lectins, fucose binding lectins, rhamnose binding lectins, galactose-binding lectins, GlcNAc-specific lectin, and GalNAc-specific lectins. Among, this C-type and mannose-binding lectins were discovered in $M$. rosenbergii.

C-type lectin is one of the proteins that is well studied in many invertebrates and constitutes common structural features such as a carbohydrate recognition domain (CRD), disulfide bond sites and calcium binding sites. The CTL reported in $M$. rosenbergii has a single or double CRD, a signal peptide that may act like a secretory protein that participates in transporting mature proteins outside the cell, a cysteine residue that forms disulfide bridges, and calcium binding sites. The CRD structure is composed of a double loop composed of four conserved cysteine residues and stabilized by two disulfide bridges. However, some CTL is present without signal peptides that may act as receptors or calcium-independent binding sites. The position of the hydrogen bond from donor and acceptors site on the Ca2+ binding site regulates the binding of carbohydrate. The CRD fold is formed by a double loop structure that is stabilized by two conserved disulfide bridges along with hydrophobic and polar interactions. CRD that has a Glu-Pro-Asn (EPN) motif binds to mannose whereas CRD that bears at GIn-ProAsp (QPD) motif binds to galactose. The Trp-Asn-Asp (WND) motif shows a connection with monosaccharide residues in the presence of the $\mathrm{Ca} 2+$ binding site. This variation shows CRD binding capability towards different sugar moieties on invading pathogens (Zelensky and Gready, 2005).

Though of CTL found in higher levels in invertebrates with diverse functionality, it is highly conserved in vertebrates. Many types of CTL's were found in Drosophila melanogaster, Caenorhabditis elegans, also in Manduca sexta that help in activation of other immune-related genes likely prophenoloxidase, encapsulation, and melanization. CTL is also found in many different penaeid shrimps that carry antimicrobial activity with single CRD, whereas the double CRD may act as PRR via binding to bacteria for further neutralization processes. The $M$. rosenbergii shows its possible anti-viral and antimicrobial roles upon $V$. anguillarum, V. parahaemolyticus, WSSV and spiroplasma challenge. Similarly, P. monodon, $M$. japonicas, and $L$. 
vannamei also show anti-viral and antimicrobial activity (Luo et al., 2003). M. rosenbergii CTL knockdown shows its association with activation of other types of innate immune systems such as anti-lipopolysaccharide factor (ALF) and lysosomes. It is also shown that CTL could initiate multiple signal transduction cascades upon pathogen challenge with the stimulation of other immune molecules.

CTL has even shown its robust activity to many types of pathogens like bacteria and virus. $M$. rosenbergii shows agglutination activity towards both Gram positive and Gram-negative bacteria which shows the possible association of lectin and carbohydrate component on the surface of pathogens. This similar activity was also observed in $F$. chinesis, $P$. monodon, $P$. japonicus, and $L$. vannamei. The recombinant CTL also shows its broad binding activity towards both Gram-positive bacteria like $S$. aureus, $M$. luteus, $B$. subtilis, $B$. cereus and $B$. megaterium and Gram-negative bacteria such as $A$. hydrophila, $V$. parahaemolyticus, $V$. anguillarum, V. alginolyticus and $E$. coli. This indicates its wide range response in defense mechanism against a range of pathogens.

Meanwhile, a mannose-binding lectin that was categorized as an AMP was also found in $M$. rosenbergii. Many mannose-binding lectins were identified and characterized, ranging from plants to vertebrates, invertebrates especially in insects and shrimps which indicates the evolution and diversity of mannose-binding lectin gene. $\mathrm{Mr}$. MBL is also predicted to have a signal peptide region, a CRD domain, a cysteine region and a calcium binding site similar to CTL. These cysteine residues are highly conserved and important in forming disulfide bonds. It even acts as a calcium-dependent lectin with four calcium binding sites and is composed of highly conserved 'specific carbohydrate recognition motifs' at the Tyr-Ser-Asn (YSN) and Gly-AspLeu (GDL) sites. The tertiary structure of this molecule is composed of $\alpha$-helix, coil regions, and $\beta$-sheets (Antony et al., 2016).

Antimicrobial activities of $\mathrm{MBL}$ were also observed in response to different bacteria such as $A$. hydrophila, E. coli, V. parahaemolyticus, V. alginolyticus, V. harveyi, B. subtilis, B. licheniformis, B. coagulans, S. pyogenes, M. luteus and L. monocytogenes. Among which, the Gram-negative bacteria show a better binding activity compared to Grampositive bacteria which may be due to the binding capacity of mannose and the carbohydrate from both bacteria. A similar result was observed by since LPS is the main pathogen associated with molecular patterns in Gramnegative bacteria. The gene expression studies show that mannose-binding lectin can be regulated upon WSBV, MrNV, A. hydrophila and V. harveyi infection. Previous reports show similar results for lectin response to Vibrio challenge in $\mathrm{M}$. rosenbergii and Fenneropenaues chinesis (Zhang et al., 2009; Xu et al., 2010). Moreover, the bactericidal efficiency test shows the possibility of killing viral and bacterial colonies efficiently. This shows that MrMBL also takes part in defense mechanism towards pathogen eradication.

\section{Apoptosis pathway}

Apoptosis is genetically controlled by the cell death program. This pathway will eliminate harmful cells (such as a viral cell) and damaged cells. This pathway is very important in some of the normal processes such as the development of the embryo, metamorphosis and more importantly in immune defense (Wen et al., 2012). Apoptosis pathway plays an important key role in crustacean immune defense against viral attacks. There are many viruses with different strategies to inhibit apoptosis in host cells during viral infections. The virus will try to delay the host cell capability till sufficient offspring viruses have been produced (Wang et al., 2008). Even though the viruses always try to inhibit apoptosis, there is an exception in some viruses that they try to use apoptosis pathway to spread their progeny virus to other cells. The most important molecules which mediate the apoptosis pathways are caspase (Elmore, 2007). Caspase overexpression was diagnosed in M,japonicas under WSSV infection (Jiann and Lo, 2011).

\section{Plasma clotting protein}

Blood loose prevention is an essential immune process in case of injury. This mechanism is different in different crustacean species. Lipoprotein is another clotting protein which is found in freshwater crayfish (Hall et al., 1999 ). Each subunit of lipoprotein has lysine and glutamine side chain. Then the injured tissue or hemocyte will release transglutaminase which will cause the chains to cross-link to each other covalently. All these reactions will happen in the presence of Ca2+ (Laszlo and Graham, 2003) as shown in Figure 1.

In some species such as crayfish, the hemolymph clotting is based on the direct transglutaminase-mediated crosslinkage of specific plasma protein. This protein is a homologous protein to the female-specific vitellogenins (Hall et al., 1999). An interesting fact about clotting protein is the similarity sequence with female-specific protein, vitellogenin, in both sexes which constitute a separate group of clotting factors. Therefore, the crustacean clotting proteins are evolutionarily related to vitellogenins, but they have completely-different functions-and are expressed in both sexes. So, they should not be considered as true vitellogenins (Lee et al., 2008).

\section{Conclusion}

Temperature is a-critical-abiotic factor that affects organisms thoroughly from the-ecological macroenvironment to the fundamental cellular and internal molecular environments. Generally, all microorganisms experience temperature fluctuations during day and night and also by seasonal changes in their natural habitat. There is a strong monitoring evidence that global temperature has been increasing in the past decades. The evidence also confirms that it will continue to increase in the future as well (Hornbach et al., 2016). The question of how organisms can be affected by global warming and climate changes has been argued since 1999, and it is still heavily concerning to humankind (Hughes et al., 2003; Sagarin et al., 1999; Stillman, 2003). 
Providing the molecular response-repertoire derived from environmental changes is essential to develop-strategies to prevent mortalities-related to climate parameter in the natural environment and in aquaculture farms. In this study, through the essential pathway of gene expression analysis, we tried to identify the resistant-related gene expression signatures in invertebrate under temperature changes. To understand the-mechanisms that underlie resistance in invertebrates, some studies have been conducted to identify gene regulations by environmental changes.

In conclusion, the innate immune system is the primordial immune system which has been evolving until today in every living system. According to a recent study, invertebrates were classified as ancestors to insects in the group of invertebrates since they could survive from a wide range of environmental effects. Hence, $M$. rosenbergii which possesses innate immunity as one of its defense systems was discussed in this review. To get a virtuous view of an important defense mechanism of $M$. rosenbergii from the context of a humoral peptide, this review focused mainly on important antimicrobial peptides, lectin, and prophenoloxidase that are being studied to date and their reactions under adverse environmental condition. The discovery of these genes in different invertebrates could help further research in applications to develop natural antibiotics or therapeutic agents which could further ensure the healthy invertebrate cultivation and maintain food security for human consumption.

\section{Acknowledgments}

We would like to acknowledge the HIR-MOHE funding H-23001G000006which was awarded to Dr. Subha Bhassu and also for postgraduate funding awarded to Ms. Tahereh Alinejad.

\section{References}

Adachi K., Hirata T. and Nishioka T. (2003), Hemocyte components in crustaceans convert hemocyanin into a phenoloxidase-like enzyme, Comparative Biochemistry and Physiology Part B: Biochemistry and Molecular Biology, 134.

Agundis C., Pereyra A. and Zenteno R. (2000), Quantification of lectin in freshwater prawn (Macrobrachium rosenbergii) hemolymph by ELISA, Comparative Biochemistry and Physiology Part B: Biochemistry and Molecular Biology, 127.

Amina A., Kamel K. (2019), Water quality of the Kebir watershed, Northeast of Algeria, Journal Clean Was, 3, 28-32.

Amparyup P., Charoensapsri W. and Tassanakajon A. (2013), Prophenoloxidase system and its role in shrimp immune responses against major pathogens. Fish \& Shellfish Immunology, 134(4), 990-1001

Amparyup P., Donpudsa S. and Tassanakajon A. (2008), Shrimp single WAP domain (SWD)-containing protein exhibits proteinase inhibitory and antimicrobial activities. Developmental \& Comparative Immunology, 32(12), 14971509

Amparyup P., Sutthangkul J., and Charoensapsri W. (2012), Pattern recognition protein binds to lipopolysaccharide and $\beta$ 1, 3-glucan and activates shrimp prophenoloxidase system. Journal of Biological Chemistry, 287(13), 10060-10069

Antony S.P., Singh I.B. and Sudheer N.S. (2011), Molecular characterization of a crustin-like antimicrobial peptide in the giant tiger shrimp, Penaeus monodon, and its expression profile in response to various immunostimulants and challenge with WSSV. Immunobiology, 216(1-2), 184-194.

Antony, J.P., Schrama J.W. and Kaushik S.J. (2016), Mineral requirements of fish: a systematic review. Reviews in Aquaculture, 8(2), 172-219.

Arasu A. and Kumaresan V. (2013). Fish lily type lectin-1 contains $\beta$-prism architecture: Immunological characterization, Molecular Immunology, 56.

Arockiaraj J., Easwvaran S., and Vanaraja P. (2012), Prophenoloxidase activating enzyme-III from giant freshwater prawn Macrobrachium rosenbergii: characterization, expression and specific enzyme activity, Molecular Biology Reports, 39(2), 1377-1386.

Arockiaraj J., Easwvaran S., Vanaraja P., Singh A., Othman R.Y., and Bhassu S. (2012). Prophenoloxidase activating enzyme-III from giant freshwater prawn Macrobrachium rosenbergii: characterization, expression and specific enzyme activity. Molecular Biology Reports, 39, 10. doi:10.1007/s11033-0110872-5

Arockiaraj J., Gnanam A.J., and Muthukrishnan D. (2013), Crustin, a WAP domain containing antimicrobial peptide from freshwater prawn Macrobrachium rosenbergii: immune characterization. Fish \& Shellfish Immunology, 34(1), 109-118

Arockiaraj J., Kumaresan V., Bhatt P., Palanisamy R. and Gnanam A.J. (2014), A novel single-domain peptide, anti-LPS factor from prawn: Synthesis of peptide, antimicrobial properties and complete molecular characterization, Peptides, 53, 7988.

Arockiaraja J., Easwvarana S., Vanarajaa P., Singhb A., Othmana R.Y. and Bhassua S. (2011), Prophenoloxidase activating enzyme-III from giant freshwater prawn Macrobrachium rosenbergii: Characterization, expression and specific enzyme activity. Molecular Biology Reporter, accepted for review.

Arockiaraja J., Easwvarana S., Vanarajaa P., Singhb A., Othmana R.Y. and Bhassua S. (2012), Effect of infectious hypodermal and haematopoietic necrosis virus (IHHNV) infection on caspase $3 c$ expression and activity in freshwater prawn Macrobrachium rosenbergii, Fish \& Shellfish Immunology, 32, 161-169.

Arockiaraja J., Puganeshwaran V., Sarasvathi E., Arun S., Tahereh A., Yasmin O.R. and Subha B. (2011), Gene profiling and characterization of arginine kinase-1 (MrAK-1) from freshwater giant prawn (Macrobrachium rosenbergii), Fish \& Shellfish Immunology, 31.

Asadullah N., Faizan A. and Farva K. (2018), Evaluation of low cost environment friendly natural extracts for the purification of drinking water, Earth Sciences Pakistan, 2, 23-25.

Asano N., Yamashita T. and Yasuda K. (2001), Polyhydroxylated alkaloids isolated from mulberry trees (Morus alba L.) and silkworms (Bombyx mori L.), Journal of Agricultural and Food Chemistry, 49(9), 4208-4213.

AsPAN A.N., Huang T.S. and Cerenius L. (1995), cDNA cloning of prophenoloxidase from the freshwater crayfish Pacifastacus leniusculus and its activation, Proceedings of the National Academy of Sciences, 92(4), 939-943.

Bartlett T.C., Cuthbertson B.J. and Shepard E.F. (2002), Crustins, homologues of an 11.5-kDa antibacterial peptide, from two species of penaeid shrimp, Litopenaeus vannamei and Litopenaeus setiferus. Marine Biotechnology, 4(3), 278-293. 
Birch-Machin I., Gao S., Huen D., McGirr R., White R. A. and Russell S. (2005), Genomic analysis of heat-shock factor targets in Drosophila, Genome Biology, 6, R63.

Brockton V., Hammond J.A. and Smith V.J. (2007), Gene characterisation, isoforms and recombinant expression of carcinin, an antibacterial protein from the shore crab, Carcinus maenas, Molecular immunology, 44(5), 943-949.

Brokordt K., Pérez H., Herrera C. and Gallardo A. (2015), Reproduction reduces HSP70 expression capacity in Argopecten purpuratus scallops subject to hypoxia and heat stress, Aquatic Biology, 23, 265-274.

Cerenius L. and Soderhall K. (2004), The prophenoloxidaseactivating system in invertebrates, Immunological Reviews, 198.

Cerenius L., Lee B.L. and Söderhäll K. (2008), The proPO-system: pros and cons for its role in invertebrate immunity, Trends in Immunology, 29(6), 263-271.

Christie A.E., Rus S. and Goiney C.C. (2007), Identification and characterization of a cDNA encoding a crustin-like, putative antibacterial protein from the American lobster Homarus americanus, Molecular Immunology, 44(13), 3333-3337.

Christophides G.K., Zdobnov E. and Barillas-Mury C. (2002), Immunity-related genes and gene families in Anopheles gambiae. Science, 298(5591), 159-165.

Cuculescu M., Hyde D. and Bowler K. (1998), Thermal tolerance of two species of marine crab, Cancer pagurus and Carcinus maenas, Journal of Thermal Biology, 23, 107-110.

Cui L., Luckhart S. and Rosenberg R. (2000), Molecular characterization of a prophenoloxidase CDNA from the malaria mosquito Anopheles stephensi, Insect Molecular Biology, 9(2), 127-137.

Dai Z.X., Zhao Z.Y. and Yin Z.X. (2009), A novel C-type lectin from the shrimp Litopenaeus vannamei possesses anti-white spot syndrome virus activity. Journal of Virology, 83(1), 347-356.

Dietz T.J. (1994). Acclimation of the threshold induction temperatures for 70-kDa and 90-kDa heat shock proteins in the fish Gillichthys mirabilis. Journal of Experimental Biology, 188, 333-338.

Elmore S. (2007). Apoptosis: A review of programmed cell death. Toxicologic Pathology, 35, 495-516. doi:10.1080/01926230701320337

Fink A.L. (1999). Chaperone-mediated protein folding. Physiological Reviews, 79, 425-449.

Fujimoto K., Okino N. and Kawabata S.I. (1995), Nucleotide sequence of the cDNA encoding the proenzyme of phenol oxidase A1 of Drosophila melanogaster, Proceedings of the National Academy of Sciences, 92(17), 7769-7773.

Fujita T. (2002). Evolution of the lectin-complement pathway and its role in innate immunity. Nature Reviews Immunology, 2, 346-353. doi:10.1038/nri800

Gorman M.J., Dittmer N.T., Marshall J.L., Marshall J.L. and Kanost M.R. (2008), Characterization of the multicopper oxidase gene family in Anopheles gambiae, Insect Biochemistry and Molecular Biology, 38, 817-824.

Gupta S., Wang Y. and Jiang H. (2005), Manduca sexta prophenoloxidase (proPO) activation requires proPOactivating proteinase (PAP) and serine proteinase homologs (SPHs) simultaneously. Insect Biochemistry and Molecular Biology, 35(3), 241-248.

Hahn J.-S., Hu Z., Thiele D.J. and Iyer V.R. (2004), Genome-wide analysis of the biology of stress responses through heat shock transcription factor. Molecular and Cellular Biology, 24, 52495256.

Hall M., Scott T. and Sugumaran M. (1995), Proenzyme of Manduca sexta phenol oxidase: purification, activation, substrate specificity of the active enzyme, and molecular cloning. Proceedings of the National Academy of Sciences, 92(17), 7764-7768.

Hall M., Wang R., van Antwerpen R., Sottrup-Jensen L. and Söderhäll K. (1999), The crayfish plasma clotting protein: A vitellogenin-related protein responsible for clot formation in crustacean blood, Proceedings of the National Academy of Sciences, USA, 96, 1965-1970.

Haroon R., Arslan C. and Shahbaz N.K. (2018), Wastewater irrigation, its impact on environment and health risk assessment in Peri urban areas of Punjab Pakistan - A review. Environmental Contaminants Reviews, 1, 30-35.

Hauton C., Brockton V. and Smith V.J. (2006), Cloning of a crustinlike, single whey-acidic-domain, antibacterial peptide from the haemocytes of the European lobster, Homarus gammarus, and its response to infection with bacteria, Molecular Immunology, 43(9), 1490-1496.

Hauton C., Hammond J.A. and Smith V.J. (2005). Real-time PCR quantification of the in vitro effects of crustacean immunostimulants on gene expression in lobster (Homarus gammarus) granular haemocytes, Developmental \& Comparative Immunology, 29(1), 33-42.

Hochachka P.W. and Somero G.N. (2002), Biochemical adaptation: mechanism and process in physiological evolution, Oxford University Press.

Hoffmann J.A. (2003), The immune response of Drosophila, Nature, 426, 33-38.

Hofmann G.E., Lund S.G., Place S.P. and Whitmer A.C. (2005), Some like it hot, some like it cold: the heat shock response is found in New Zealand but not Antarctic notothenioid fishes, Journal of Experimental Marine Biology and Ecology, 316, 7989.

Hopkin R.S., Qari S., Bowler K., Hyde D. and Cuculescu M. (2006), Seasonal thermal tolerance in marine Crustacea, Journal of Experimental Marine Biology and Ecology, 331, 74-81.

Hornbach M.J., Richards M., Blackwell D., Mauroner C. and Brokaw C. (2016), 40 years of surface warming in the Northern US rocky mountains: Implications for Snowpack Retreat, American Journal of Climate Change, 5, 275.

Hosseini S.A. and Sharifi F. (2019), Improvement stability of earth canal banks using geo-technical approaches, Geology, Ecology, and Landscapes, 3, 308-317. DOI: 10.1080/24749508.2018.1563751.

Huang L.H., Christensen B.M. and Chen C.C. (2001), Molecular cloning of a second prophenoloxidase CDNA from the mosquito Armigeres subalbatus: prophenoloxidase expression in blood-fed and microfilariae-inoculated mosquitoes, Insect Molecular Biology, 10(1), 87-96.

Hughes A.L. (1999), Evolution of the arthropod prophenoloxidase/hexamerin protein family, Immunogenetics, 49.

Hughes T.P., Baird A.H., Bellwood D.R., Card M., Connolly S.R., Folke C., . . . Kleypas J. (2003). Climate change, human impacts, and the resilience of coral reefs. Science, 301, 929933.

Imjongjirak C., Amparyup P. and Tassanakajon A. (2007), Antilipopolysaccharide factor (ALF) of mud crab Scylla 
paramamosain: molecular cloning, genomic organization and the antimicrobial activity of its synthetic LPS binding domain, Molecular Immunology, 44(12), 3195-3203.

Imjongjirak C., Amparyup P. and Tassanakajon A. (2009), Molecular cloning and characterization of crustin from mud crab Scylla paramamosain, Molecular Biology Reports, 36(5), 841-850.

Imjongjirak C., Amparyup P. and Tassanakajon A. (2011), Two novel antimicrobial peptides, arasin-likeSp and GRPSp, from the mud crab Scylla paramamosain, exhibit the activity against some crustacean pathogenic bacteria, Fish \& Shellfish Immunology, 30(2), 706-712.

Islam M.A., Islam S.M.A. and Sathi M.A. (2020), Identification of lentil varieties/lines resistant to stemphylium blight considering disease reaction and yield. Malaysian Journal of Sustainable Agriculture, 4, 22-25.

Iwanaga S., and Morita T. (1978), Chromogenic substrates for horseshoe crab clotting enzyme, Haemostasis, 7, 183-188. Doi:10.1159/000214260

Jang I.K., Pang Z., Yu J., Kim S.K., Seo H.C. and Cho Y.R. (2011), Selectively enhanced expression of prophenoloxidase activating enzyme 1 (PPAE1) at a bacteria clearance site in the white shrimp, Litopenaeus vannamei, BMC Immunology, 1270.

Jesu Costa Ferreira J. and Conserva L.M. (2012), Borreria and Spermacoce species (Rubiaceae): A review of their ethnomedicinal properties, chemical constituents, and biological activities, Pharmacognosy Reviews, 6(11), 46.

Jiann H.L. and Lo C.-F. (2011), A review of the strategies evolved by WSSV to thwart host responses to infection and ensure successful virus replication in cells - apoptosis and antiapoptosis strategies. Diseases in Asian Aquaculture.

Jiravanichpaisal P., Lee S.Y. and Andrén T. (2007), Antibacterial peptides in hemocytes and hematopoietic tissue from freshwater crayfish Pacifastacus leniusculus: characterization and expression pattern, Developmental \& Comparative Immunology, 31(5), 441-455.

Junprung W., Supungul P. and Tassanakajon A. (2017), HSP70 and HSP9O are involved in shrimp Penaeus vannamei tolerance to AHPND-causing strain of Vibrio parahaemolyticus after nonlethal heat shock, Fish \& shellfish immunology, 60, 237-246.

Kilpatrick D.C. (2002), Animal lectins: a historical introduction and overview, Biochimica et Biophysica Acta (BBA)-General Subjects, 1572(2-3), 187-197.

Ko C.-F., Chiou T.-T. and Vaseeharan B. (2007), Cloning and characterisation of a prophenoloxidase from the haemocytes of mud crab Scylla serrata, Developmental and Comparative Immunology, 31, 12-22.

Ko C.F., Chiou T.T. and Vaseeharan B. (2007), Cloning and characterisation of a prophenoloxidase from the haemocytes of mud crab Scylla serrata. Developmental \& Comparative Immunology, 31(1), 12-22.

Ko C.-F., Chiou T.-T., Vaseeharan B., Lu J.-K. and Chen J.-C. (2007), Cloning and characterisation of a prophenoloxidase from the haemocytes of mud crab Scylla serrata, Developmental and Comparative Immunology, 31, 12-22.

Lee K.W. and Hwang D.S. (2008). Molecular cloning, phylogenetic analysis and developmental expression of a vitellogenin (Vg) gene from the intertidal copepod Tigriopus japonicus, Comparative Biochemistry and Physiology Part B: Biochemistry and Molecular Biology, 150(4), 395-402.
Li Y., Wang Y. and Jiang H. (2009), Crystal structure of Manduca sexta prophenoloxidase provides insights into the mechanism of type 3 copper enzymes, Proceedings of the National Academy of Sciences, 106(40), 17002-17006.

Li Y., Wang Y. and Jiang H. (2009), Crystal structure of Manduca sexta prophenoloxidase provides insights into the mechanism of type 3 copper enzymes, Proceedings of the National Academy of Sciences of the United States of America, 106(40), 17002-17006.

LI Y.-H., Zheng F.-L. and Chen H.-Q. (2009), Cloning and Sequence Analysis of Prophenoloxidase from Haemocytes of the Red Swamp Crayfish, Procambarus clarkia, Agricultural Sciences in China, 8, 369-379.

Lindquist S. (1986), The heat-shock response. Annual review of biochemistry, 55, 1151-1191.

Liu B., Deikus G. and Bree A. (2014), Global analysis of mRNA decay intermediates in Bacillus subtilis wild-type and polynucleotide phosphorylase-deletion strains, Molecullar Microbiology, 94(1), 41-55.

Liu C.H., Cheng W. and Hsu J.P. (2004), Vibrio alginolyticus infection in the white shrimp Litopenaeus vannamei confirmed by polymerase chain reaction and $16 \mathrm{~S}$ rDNA sequencing, Diseases of Aquatic Organisms, 61(1-2), 169174.

Liu H., Jiravanichpaisal P., Söderha I., Cerenius L. and Soderha K. (2006), Antilipopolysaccharide Factor interferes with white spot syndrome virus replication in vitro and in vivo in the crayfish Pacifastacus leniusculus, Journal of Virological Methods, 80, 10365-10371.

Lorand L. and Graham R.M. (2003), Transglutaminases: crosslinking enzymes with pleiotropic functions. Nature Reviews Molecular Cell Biology, 4(2), 140-156.

Lu C., Barr D.B. and Pearson M. (2006), A longitudinal approach to assessing urban and suburban children's exposure to pyrethroid pesticides, Environmental Health Perspectives, 114(9), 1419-1423.

Lu K.Y., Huang Y.T. and Lee H.H. (2006), Cloning the prophenoloxidase cDNA and monitoring the expression of proPO mRNA in prawns (Macrobrachium rosenbergii) stimulated in vivo by $\mathrm{CpG}$ oligodeoxynucleotides, Fish \& Shellfish Immunology, 20(3), 274-84.

Luo T., Zhang X., Shao Z. and Xub X. (2003), PmAV, a novel gene involved in virus resistance of shrimp Penaeus Monodon FEBS Letters, 551, 53-57.

Madeira D. (2014), Role of thermal niche in the cellular response to thermal stress: Lipid peroxidation and HSP70 expression in coastal crabs, Ecological indicators, 36(2036), 601-606. doi:10.1016/j.ecolind.2013.09.023

Marques M.R. and Barracco M.A. (2000), Lectins, as non-selfrecognition factors, in crustaceans, Aquaculture, 191.

McGreal E.P., Martinez-Pomares L. and Gordon S. (2004), Divergent roles for C-type lectins expressed by cells of the innate immune system, Molecular Immunology, 41(11), 1109-1121.

Morimoto R.I. and Santoro M.G. (1998), Stress-inducible responses and heat shock proteins: new pharmacologic targets for cytoprotection, Nature biotechnology, 16, 833.

Morita T., Ohtsubo S. and Nakamura T. (1985), Isolation and Biological Activities of Limulus Anticoagulant (AntiLPS Factor) which Interacts with Lipopolysaccharide (LPS), The Journal of Biochemistry, 97(6), 1611-1620. 
Nagoshi H., Inagawa H. and Morii K. (2006), Cloning and characterization of a LPS-regulatory gene having an LPS binding domain in kuruma prawn Marsupenaeus japonicus, Molecular Immunology, 43, 2061-2069.

Nayak S., Singh S. and Ramaiah N. (2010), Identification of upregulated immune-related genes in Vibrio harveyi challenged Penaeus monodon postlarvae, Fish \& Shellfish Immunology, 29(3), 544-549.

Ogunyele A.C., Obaje S.O. and Akingboye A.S. (2018), Lithostructural relationships and petrogenetic affinities of the basement complex rocks around Okpella, Southwestern Nigeria, Earth Sciences Malaysia, 2, 29-36.

Oren A., Ventosa A. and Nieto J.J. (1998), Biology of moderately halophilic aerobic bacteria, Microbiology and molecular biology reviews, 62(2), 504-544.

Parsell D. and Lindquist S. (1993), The function of heat-shock proteins in stress tolerance: degradation and reactivation of damaged proteins, Annual review of genetics, 27, 437-496.

Pereyra A., Zenteno R. and Vázquez L. (2004), Characterization of lectin aggregates in the hemolymph of freshwater prawn Macrobrachium rosenbergii, Biochimica et Biophysica Acta (BBA)-General Subjects, 1673.

Ranganathan P., Subramanian A. and Diamond S.L. (1999), Nuclear targeting peptide scaffolds for lipofection of nondividing mammalian cells, Nature Biotechnology, 17(9), 873-877.

Rattanachai A., Hirono I. and Ohira T. (2004), Molecular cloning and expression analysis of $\alpha 2$-macroglobulin in the kuruma shrimp, Marsupenaeus japonicus, Fish \& Shellfish Immunology, 16(5), 599-611.

Ravaux J., Léger N., Rabet N., Fourgous C., Voland G., Zbinden M. and Shillito B. (2016), Plasticity and acquisition of the thermal tolerance (upper thermal limit and heat shock response) in the intertidal species Palaemon elegans, Journal of Experimental Marine Biology and Ecology, 484, 39-45.

Relf J.M. and Chisholm J.R. (1999), Purification and characterization of a cysteine-rich 11.5-kDa antibacterial protein from the granular haemocytes of the shore crab, Carcinus maenas, European Journal of Biochemistry, 264(2), 350-357.

Relf J.M., Chisholm J.R. and Kemp G.D. (1999). Purification and characterization of a cysteine-rich $11.5-\mathrm{kDa}$ antibacterial protein from the granular haemocytes of the shore crab, Carcinus maenas. European Journal of Biochemistry.

Ren Q., Zhang Z. and Hui K.M. (2012), Three different antilipopolysaccharide factors identified from giant freshwater prawn, Macrobrachium rosenbergii, Fish \& Shellfish Immunology, 33(4), 766-774.

Ritossa F. (1962), A new puffing pattern induced by temperature shock and DNP in Drosophila, Experientia, 18, 571-573.

Rosa R.D. and Barracco M.A. (2010), Antimicrobial peptides in crustaceans, Invertebrate Survival Journal, 7, 262-284.

Rosa R.D., Bandeira P.T. and Barracco M.A. (2007), Molecular cloning of crustins from the hemocytes of Brazilian penaeid shrimps, FEMS Microbiology Letters, 274(2), 287-290.

Sagarin R.D., Barry J.P., Gilman S.E. and Baxter C.H. (1999), Climate-related change in an intertidal community over short and long time scales, Ecological Monographs, 69, 465-490.

Sharon N. and Lis H. (1998), Lectins: Carbohydrate-Specific proteins that mediate cellular recognition, Chemical Reviews, 98.
Smith V.J., Brown J.H. and Hauton C. (2003), Immunostimulation in crustaceans: does it really protect against infection?, Fish \& Shellfish Immunology, 15, 71-90.

Söderhall I., Bangyeekhun E., Mayo S. and Söderhall K. (2003). Hemocyte production and maturation in an invertebrate animal; proliferation and gene expression in hematopoietic stem cells of Pacifastacus leniusculus, Developmental and Comparative Immunology, 27, 661-672.

Söderhäll K. and Cerenius L. (1998), Role of the prophenoloxidaseactivating system in invertebrate immunity, Current Opinion in Immunology, 10(1), 23-28.

Somboonwiwat K., Bachère E. and Rimphanitchayakit V. (2008), Localization of anti-lipopolysaccharide factor (ALFPm3) in tissues of the black tiger shrimp, Penaeus monodon, and characterization of its binding properties, Developmental \& Comparative Immunology, 32(10), 1170-1176.

Stillman J.H. (2003), Acclimation capacity underlies susceptibility to climate change, Science, 301, 65-65.

Stoss T.D., Nickell M.D. and Hardin D. (2004), Inducible transcript expressed by reactive epithelial cells at sites of olfactory sensory neuron proliferation, Journal of Neurobiology, 58(3), 355-368.

Sugumaran M. and Nellaiappan K. (1996), On the presence of prophenoloxidase in the hemolymph of the horseshoe crab, Limulus, Comparative Biochemistry and Physiology Part B: Biochemistry and Molecular Biology, 113.

Sun N., Qin Y. and Rogers R.D. (2010), Dissolution or extraction of crustacean shells using ionic liquids to obtain high molecular weight purified chitin and direct production of chitin films and fibers, Green Chemistry, 12(6), 968-971.

Supungul P., Klinbunga S. and Pichyangkura R. (2002), Identification of immune-related genes in hemocytes of black Tiger Shrimp (Penaeus monodon), Journal of Marine Biotechnology, 4, 487.

Supungul P., Klinbunga S. and Pichyangkura R. (2004), Antimicrobial peptides discovered in the black tiger shrimp Penaeus monodon using the EST approach, Diseases of Aquatic Organisms, 61(1-2), 123-135.

Supungul P., Tang S. and Maneeruttanarungroj C. (2008), Cloning, expression and antimicrobial activity of crustinPm1, a major isoform of crustin, from the black tiger shrimp Penaeus monodon, Developmental and Comparative Immunology, 32, 61-70.

Suzuki K., Suzuki I., Leodolter A., Alonso S., Horiuchi S., Yamashita K. and Perucho M. (2006), Global DNA demethylation in gastrointestinal cancer is age dependent and precedes genomic damage, Cancer Cell, 9, 199-207.

Taft A.S., Chen C.C. and Christensen B.M. (2001), Molecular cloning of two prophenoloxidase genes from the mosquito Aedes aegypti, Insect Molecular Biology, 10(1), 97-103.

Tharntada S., Ponprateep S. and Somboonwiwat K. (2009), Role of anti-lipopolysaccharide factor from the black tiger shrimp, Penaeus monodon, in protection from white spot syndrome virus infection, Journal of General Virology, 90(6), 1491-1498.

Tharntada S., Somboonwiwat K. and Rimphanitchayakit V. (2008), Anti-lipopolysaccharide factors from the black tiger shrimp, Penaeus monodon, are encoded by two genomic loci, Fish \& Shellfish Immunology, 24(1), 46-54.

Theopold U., Schmidt O. and Söderhäll K. (2004), Coagulation in arthropods: defence, wound closure and healing, Trends in Immunology, 25(6), 289-294. 
Tomanek L., and Somero G.N. (1999), Evolutionary and acclimation-induced variation in the heat-shock responses of congeneric marine snails (genus Tegula) from different thermal habitats: implications for limits of thermotolerance and biogeography, Journal of Experimental Biology, 202, 2925-2936.

Underhill D. and Ozinsky A. (2002), Toll-like receptors: key mediators of microbe detection. Current Opinion in Immunology, 14, 103-110.

Vazquez L., Alpuche J., Maldonado G., Agundis C., PereyraMorales A. and Zenteno E. (2009), Immunity mechanisms in crustaceans, Innate Immunity, 15, 179-188.

Vázquez-Mendoza A., Carrero J.C. and Rodriguez-Sosa M. (2013), Parasitic infections: a role for $\mathrm{C}$-type lectins receptors, BioMed Research International.

Wang L., Zhang Y. and Gao J. (2011), AlF 3 coating of LiNi $0.5 \mathrm{Mn}$ 1.504 for high-performance Li-ion batteries, lonics, 17(8), 671.

Wang L., Zhi B., Wu W. and Zhang X. (2008), Requirement for shrimp caspase in apoptosis against virus infection, Developmental \& Comparative Immunology, 32, 706-715.

Wen R., Li F., Xie Y., Li S., and Xiang J. (2012), A homolog of the cell apoptosis susceptibility gene involved in ovary development of Chinese shrimp Fenneropenaeus, Biology of Reproduction, 86, 1-7.

Xu Y. and Liu H. (2010), A polymorphism in the microRNA-30e precursor associated with major depressive disorder risk and P300 waveform, Journal of Affective Disorders, 127(1-3), 332-336.

Yang S.P., Wu Z.H. and Jian J.C. (2010). Effect of marine red yeast Rhodosporidium paludigenum on growth and antioxidant competence of Litopenaeus vannamei. Aquaculture.

Youdeowei P.O., Nwankwoala H.O. and Desai D.D. (2019). Dam structures and types in nigeria: sustainability and effectiveness, Water Conservation and Management, 3, 2026.

Yue F., Pan L. and Xie P. (2010), Immune responses and expression of immune-related genes in swimming crab Portunus trituberculatus exposed to elevated ambient ammonia- $\mathrm{N}$ stress, Comparative Biochemistry and Physiology Part A: Molecular \& Integrative Physiology, 157(3), 246-251.

Zelensky A.N. and Gready J.E. (2005), The C-type lectin-like domain superfamily, The FEBS Journal, 272(24), 6179-6217.

Zhang X., Jiang H. and Wang Q. (2009), Chasing relationships between nutrition and reproduction: $A$ comparative transcriptome analysis of hepatopancreas and testis from Eriocheir sinensis, Comparative Biochemistry and Physiology Part D: Genomics and Proteomics, 4.

Zhang X.J., Liu Y.C. and Dong B. (2007), Molecular cloning, characterization and expression analysis of a putative C-type lectin (Fclectin) gene in Chinese shrimp Fenneropenaeus chinensis, Molecular Immunology, 44(4), 598-607.

Zhao D., Song S., Wang Q., Zhang X., Hu S. and Chen L. (2009). Discovery of immune-related genes in Chinese mitten crab (Eriocheir sinensis) by expressed sequence tag analysis of haemocytes, Aquaculture, 287, 297-303.

Zulkapli M.F., Rashid N.M., Mohd Nazri M.S. and Noorshawal N. (2018), Study on optical properties of graphene-Tio 2 nanocomposite as photoanodes layer in dye sensitized solar cell (Dssc), Environment \& Ecosystem Science, 2, 39-41. 